\title{
The unexpected diversity of plant organelle RNA editosomes
}

Tao Sun ${ }^{1,2}$, Stephane Bentolila ${ }^{1}$, Maureen Hanson ${ }^{1 *}$

\section{Keywords}

chloroplasts, mitochondria, protein complex, pentatricopeptide, RNA recognition motif, zinc finger motif 
19 Flowering plants convert many hundreds of organelle Cs to Us during post-transcriptional RNA editing. Pentatricopeptide Repeat (PPR) proteins dictate specificity by recognizing RNA sequences near $C$ targets. However, the complete mechanism of the editing machinery is not yet understood. Recently, non-PPR editing factors (RIP/MORFs, ORRMs, OZ and PPO1) have been identified as components of the plant RNA editosome, which is a small RNA/protein complex. Surprisingly, plant editosomes are highly diverse with regard not only to the PPR proteins they contain, but also in the non-PPR components that are present. Here we review the most recent progress in the field and discuss the implications of the diversity of plant editosomes for evolution of RNA editing and for possible future applications.

Plant RNA editing- a correction mechanism with high specificity

Once believed to be merely an apprentice of DNA, RNA is now known to undergo RNA editing, a process that differentiates the RNA sequence from its DNA master [1-3]. In vascular plants, RNA editing converts many hundred cytidines (C) to uridines (U) at specific positions in plastid and mitochondrial transcripts [4-7]. Editing often restores conserved codons or creates start or stop codons, serving as a correction mechanism for otherwise defective organellar transcripts [5]. Despite some examples of unimportant editing events that are either silent or in non-coding regions, many RNA editing events have been shown to be essential for proper function of proteins and optimal growth of plants [8-14].

Cytidine can be converted to uridine by a simple deamination reaction. In animals and humans, two deaminase-mediated RNA editing mechanisms, the C-to-U editing by APOBEC and A-to-I editing by ADAR, have been identified [15-24]. While APOBEC- and ADARmediated editing has been shown to create multiple proteins from differentially edited transcripts, no evidence supports the same function in plants [11, 25]. Although unedited or partially edited transcripts can load onto plant mitochondrial ribosomes, there are no examples of useful protein diversity produced by translation of incompletely edited transcripts. In two cases that have been investigated carefully, the plant proteins that accumulate are specified primarily by edited transcripts, likely due to instability of aberrant proteins resulting from translation of improperly edited transcripts [26-28]. Instead of creating protein polymorphism, plant RNA 
editing is believed to be a correction mechanism to cope with detrimental T-to-C mutations

50 inherited from the organelle genome.

51 Editing events are being gained and lost over evolutionary time. A C in one species is

52 sometimes not edited in another because a $T$ is already genomically encoded $[5,9,14]$. While

53 RNA editing would not be needed if all detrimental T-to-C mutations were changed back to Ts,

54 once many have accumulated, converting all of them becomes less likely, resulting in retention

55 of the RNA editing apparatus [29, 30]. Nevertheless, overall, plant RNA editing events are

56 gradually becoming lost; in general, lower plants and gymnosperms exhibit a larger number of

57 target sites than angiosperms $[6,31,32]$

58 Another distinct property of the plant RNA editing activity is the high specificity

59 governed by RNA-binding Pentatricopeptide Repeat (PPR) proteins [31, 33]. Human ADAR

60 editing can be rather promiscuous across many thousands of adenosine targets residing in

61 double-stranded RNAs [34]. While the human APOBEC system contains an RNA sequence

62 recognition factor, only a few targets can be specified [11, 17, 19]. In contrast, the plant editing

63 apparatus (the editosome) can specifically target hundreds of individual sites. Unlike the

64 APOBEC system, plant RNA editosomes are able to incorporate different RNA-binding proteins

65 that recognize a variety of RNA sequences near the $\mathrm{C}$ targets of editing. Vascular plants such as

66 arabidopsis (Arabidopsis thaliana) encode hundreds of PPR proteins, which provide an

67 unparalleled capacity for specific editing [31, 35].

Modular RNA/protein complexes that carry targeting specificity and enzymatic activity

70 CRISPR/Cas9 system originally found in bacteria [36-38]. Initial efforts have been made to use

71 mammalian ADAR combined with guiding RNA to correct mutations in cell lines [39]. Given its

72 novel properties, the plant RNA editing system potentially offers an alternative mechanism for

73 engineering purposes. The discovery of a recognition code for specifying the RNA sequences

74 recognized by PPR motifs have been followed by some recent examples of reprogramming PPR

75 proteins to target desired RNA sequences [33, 40-42]. All of the trans-factors needed for RNA

76 editing in chloroplasts are nuclear-encoded [43, 44]. However, unlike the CRISPR/Cas9 system,

77 to date the composition of the plant editosome is not fully understood; further application will be

78 obstructed without more information about plant editosomes. 
Starting from the discovery of RIP/MORF editing factors, great progress has been made in identifying the other protein components of the editosome, now including the ORRM and $\mathbf{O Z}$ protein families. Unexpectedly, editosomes across different targets differ not only in their PPR recognition factors but also in other components. This review will focus on the recent progress

83 in the plant editing field, with a focus on the non-PPR editing factors that point to an unexpected 84 diversity of plant editosomes.

\section{PPR proteins} Compared to merely 7 members in humans, the PPR-motif containing family has substantially increased in the plant kingdom, with over 400 members in a typical flowering plant [30, 35, 45, 46]. Each member carries multiple $\sim 35$ amino acid repeats (PPR motif) in tandem, which recognize specific cis-elements for editing in a combinatorial pattern (Figure 1A) [40, 47, 48]. The PPR proteins involved in editing belong to a subclass that has slightly longer or shorter PPR motifs and additional domains other than PPR motifs [14, 30, 46]. Two functional domains- the Extension (E) domain and the DYW domain have been identified in this subclass of PPR proteins (Figure 1A). In Arabidopsis, approximately 193 PPR members contain an E domain, while 87 contain both E and DYW domains [35]. The E domain has been shown to be essential for editing, especially the PG region [49-51].

Because the sequence of the DYW domain exhibits some similarity to cytidine deaminase domains found in other systems, it was proposed to be the catalytic factor for plant editing [52]. As well as their presence in plants, DYW domains have been discovered in protists [53]. However, while in vitro editing systems using chloroplast or mitochondrial extracts and synthetic RNA have been established [54-58], no deaminase activity has been detected through expression

102 of recombinant DYW-type PPR editing factors [55-57, 59]. Divalent cations are evidently 103 required for editing, since EDTA and EGTA severely inhibit the editing activity [55]. Notably,

104 plant editing in vitro is sensitive to zinc chelators, indicating zinc ion is an essential co-factor, 105 similar to other deaminase systems $[55,57]$.

An essential role of the DYW domain was challenged by the finding that DYW domains

107 in certain PPR proteins are dispensable for editing [51, 60]. In one case, an Arabidopsis gene 108 encoding a protein with a DYW domain, but no PPR motifs, was found to be necessary for 109 editing of a $\mathrm{C}$ target recognized by a E-type PPR protein (Figure 1A), which lacks a DYW 
110 domain [61]. This raises the possibility that the other $\mathrm{C}$ targets whose cis-elements are

111 recognized by E-type PPR proteins are also utilizing DYW-type PPR proteins to provide the

112 deaminase activity. However, examples of $\mathrm{C}$ targets that require more than one PPR protein are

113 rare [62-64], even though a number of PPR-protein-encoding genes have been subjected to

114 mutagenesis. A possible explanation is redundancy of the DYW-type PPR proteins that are able

115 to provide the enzyme activity to an editosome carrying a site-specific E-type PPR protein

116 recognition factor. In that case, even if one DYW-type PPR protein is not available due to

117 mutation, others would be able to carry out the deamination task. Alternatively, there may be

118 proteins, which are as yet unidentified, that supply catalytic activity to editosomes.

119 While deletion analysis has shown that the DYW domain is dispensable in some DYW-

120 type PPR proteins, editing activity and zinc binding is lost when the DYW domain is missing or

121 removed from certain PPR proteins [49]. Identification of PPR-DYW proteins in which the

122 DYW domain was essential allowed three different groups to examine the importance of

123 conserved residues in the DYW domains of those PPR proteins. Site-specific mutagenesis

124 within the conserved motifs of DYW domains greatly reduced editing extent $[49,61,65]$ and

125 binding of zinc, a recognized feature of known deaminases [61, 66], which is required for editing 126 in vitro $[55,58]$.

127 Until recently, all identified editing factors belonged to the PPR family [5, 14, 67]. Given

128 that PPR proteins could possibly carry both RNA binding activity and the enzymatic activity, a

129 model could be envisioned in which multimers of PPR proteins alone could constitute the

130 editosome [52]. However, this hypothesis was completely overturned by the discovery of

131 essential non-PPR protein components in the editosome-first the RIP/MORF family, then the

132 ORRM family and later a member of the OZ family (Table 1) [4, 68-73].

\section{RNA editing factor-interacting proteins lacking PPR motifs}

135 When a chloroplast extract was subjected to size exclusion chromatography, the $70 \mathrm{kDa}$ PPR

136 protein RARE1was found to be present in a small protein complex that separated between 200

137 and $443 \mathrm{kDa}$ protein markers [68]. Immunoprecipitation of an epitope-tagged RARE1 resulted in

138 the identification of one non-PPR protein that strongly interacted with RARE1-RNA editing

139 factor Interacting Protein 1 (RIP1) [68]. In a ripl hypomorph Arabidopsis mutant, editing extent

140 of over 400 editing Cs in mitochondria and 11 editing sites in chloroplasts was reduced, making 
141 it the most influential editing factor in plants yet identified [4]. Other members of the RIP1

142 family were identified as Multiple Organellar RNA editing Factors (MORFs) through genetic

143 screening for mitochondrial editing defects in a collection of Arabidopsis EMS mutants [73]. In

144 contrast to genes encoding PPR proteins, mutagenesis of a single gene encoding a RIP/MORF

145 protein affects dozens or even hundreds of editing sites. In order to analyze such large number of

146 editing targets, high-throughput methods based on next generation sequencing and mass

147 spectrometry are particularly valuable methods $[4,80]$. Of the 10 members of the RIP/MORF

148 family in Arabidopsis, 5 are major editing factors for mitochondria or/and plastids, while the

149 remaining family members have minor or no effect on editing [4]. In plastids, RIP2/MORF2 and

150 RIP9/MORF9 are major players, required for almost every editing site $[4,73]$. In mitochondria,

151 RIP1/MORF8 affects over 70\% of all the editing events and RIP3/MORF3, RIP8/MORF1 affect

$15226 \%$ and $19 \%$ respectively (Table 1) $[4,68,73]$.

153 While a motif of unknown function is conserved across RIP/MORF members,

154 RIP/MORF proteins do not have any annotated domain, and some contain an extended C-

155 terminus (Figure 1B)[4, 73]. RIP/MORF proteins interact with the PPR factors that address the

156 same editing sites, via the connection to the PPR tract or other regions [68, 81]. Different

157 RIP/MORF editing factors may affect different sets of editing events, while some editing events

158 can be affected by two or more different RIP/MORFs [4]. This may be due to a requirement of a

159 homomer of RIP/MORF for some sites but a heteromer for others. Such types of interaction were

160 indeed observed among RIP/MORF members through protein-protein interaction studies (Table

161 2) $[73,81]$. The function of this family is still unknown, though speculation exists that

162 RIP/MORF proteins bridge the PPR factor and the deaminase activity in the editosome [81].

163 Like the PPR family, the RIP/MORF family is widespread among angiosperms.

164 However, genes encoding RIP/MORF proteins are missing in some ferns and moss species,

165 which also carry out organelle RNA editing $[68,73,82]$. Why angiosperm RNA editing requires

166 these proteins, but other plants do not, is a mystery; less information is available about non-PPR

167 editing factors in non-vascular plants.

168

169 Organelle RNA Recognition Motif proteins

170 Organelle RNA Recognition Motif protein1 (ORRM)1, the first ORRM editing factor, was

171 found in silico as a protein related to the RIP family; it has two truncated RIP domains in 
172 addition to an RRM domain [69]. ORRM1 controls $>60 \%$ of plastid editing sites in Arabidopsis

173 (Table1). Surprisingly, the ORRM1 RRM domain alone can restore editing to an orrm1 mutant,

174 according to a transient complementation assay [69]. A genetic screening of photosynthetic

175 mutations in a maize insertional mutant collection identified the maize ortholog of ORRM1,

176 which was confirmed as a plastid editing factor as well, implying a conserved editing function

177 across species [69]. Although the RRM is a highly common motif in eukaryotes, the motif in

178 ORRM1 belongs to a distinct clade of approximately 20 members in Arabidopsis, most of which

179 are organelle-targeted. Except for ORRM1, none of the other members carries a RIP domain.

180 Instead, many contain glycine-rich regions [69] (Figure 1C).

181 Four ORRM proteins have been reported to date to be RNA editing factors (Table 1) [69,

$18271,72,74]$. ORRM1 is a major factor in plastids, while ORRM2, ORRM3 and ORRM4 are

183 mitochondrial editing factors [74]. Similar to RIP/MORF factors, ORRMs affect many editing

184 events in a site-specific manner, though ORRM4 also broadly affects editing events in the same

185 transcripts $[69,71,72]$. ORRMs can interact with RIP/MORF factors and form homo- or

186 heterodimers [70-72]. ORRM1 associates with PPR editing factors that control the common

187 editing events, while the interaction between ORRM2-4 and PPR factors might not be direct, but

188 rather mediated via RIP/MORF or other components of the editosome (Table 2) [69, 71, 72].

189 The function of the RRM domain in editing is still an open question. ORRM1 can bind near

190 some editing cis-elements [69]. Why a plant editosome would require a second RNA binding

191 activity besides PPR factor is not clear. One speculation is that RRM binds in the vicinity of

192 editing sites, preparing the RNA for entry of additional factors or enhancing the specificity of the

193 PPR-protein mediated cis-element recognition [78]. Alternatively, instead of binding RNA,

194 perhaps some RRMs bind other protein components of the editosome.

195

196 Organelle Zinc finger proteins (OZ)

197 Analogous to the discovery of RIP1, OZ1 was found in an ORRM1 co-purified protein complex

198 [70]. Loss of $O Z 1$ in Arabidopsis leads to editing defects at 30 plastid sites, which eventually 199 results in a virescent phenotype (Table 1). Four members comprise the OZ family, all of which

200 are organelle targeted. The only annotated domain of the family is the RanBP2 type zinc finger,

201 which is repeated in various numbers in the OZ members (Figure 1D); however, there is another 202 conserved domain of unknown function in the four OZ proteins. 
OZ1 selectively interacts with the PPR specificity factors and also strongly associates

204 with ORRM1, but does not appear to directly bind RIP/MORFs. In addition, OZ1 can dimerize

205 (Table 2) [70]. The function of the zinc finger and other uncharacterized regions of the OZ

206 proteins in the editosome awaits further investigation. Plant C-to-U editing has been shown to be

207 zinc ion dependent, possibly due to the requirement of $\mathrm{Zn}^{2+}$ in the active center of cytidine

208 deaminase [58, 83]. It is not yet known whether zinc fingers in $\mathrm{OZ}$ actually bind $\mathrm{Zn}^{2+}$ and

209 whether such binding affects editing. The widespread OZ family can be detected in many plant

210 lineages, including Selaginella, suggesting an evolutionally conserved function in the plant

211 editosome [70].

212

\section{Additional factors}

214 Three additional proteins - CP31, PPO1 and OCP3--have been found to affect RNA editing

215 efficiency in plastids. Loss of CP31 leads to reduced editing levels at multiple plastid sites and

216 immunodepletion of the CP31 protein also inhibits in vitro editing of some substrates (Table 1)

217 [78]. Given that CP31 is an RRM containing protein, whether CP31 and ORRM operate in a

218 similar fashion is an intriguing question. However, CP31 does not belong to the ORRM clade,

219 nor does it affect editing in a site-specific pattern that is seen in orrml [69, 78]. Moreover,

220 transcript abundance is greatly reduced in cp31 mutant, which suggests CP31 may primarily be

221 an RNA stability factor [78, 79].

222 Protoporphyrinogen Oxidase 1 (PPO1), a key enzyme for tetrapyrrole metabolism, was

223 shown to have a surprising role in plastid RNA editing [76]. Reduced editing was observed for

22418 plastid sites when PPO1 is mutated, and such defects can be rescued by a truncated PPO1

225 protein that lacks oxidase enzymatic activity (Table 1). PPO1 directly interacts with the plastid

226 RIP/MORF proteins but not with the PPR factors (Table 2). However, except for one site, none

227 of the affected sites completely loses editing when PPO1 is absent [76]. Most of the affected

228 sites are in the $n d h$ gene transcripts

229 Overexpression of Cationic Peroxidase 3 (OCP3) affects editing of multiple sites in the

230 plastid $n d h B$ transcript (Table 1) [84]. Though ocp3 mutant exhibit only mildly reduced editing

231 extent, it seemed sufficient to impair NDH activity, which consequently enhanced the plant's

232 resistance to fungal infection [84].

233 


\section{The diversity of plant RNA editosomes}

235 RNA editing must be confined to particular Cs to prevent detrimental changes in transcripts. The 236 specificity of recognition of 5' cis-elements is clearly explained by the polypeptide code for 237 recognition of particular nucleotides by the repeat motifs [40, 47, 85-87]. Editing is often

238 maintained when a few nucleotides are altered in the cis-elements, thus allowing a few different

239 C targets carrying similar but not identical cis-elements to be recognized by a single PPR protein.

240 This flexibility in recognition site makes sense evolutionarily; as new $\mathrm{T}$ to $\mathrm{C}$ mutations arise, the

241 plant must evolve new PPR proteins for recognition, perhaps based on a protein that provides 242 weak binding and partial editing. The flexibility also explains how <200 editing-type PPR 243 proteins can specify $>600 \mathrm{C}$ targets in Arabidopsis.

244 Up to about 200 different editosomes could exist in Arabidopsis organelles, if all the

245 other components are identical. An early hypothesis was that the plant editing system consisted

246 of one or more PPR proteins that recruited a single editing activity to the proper location,

247 analogous to the RNA-binding protein that binds 3' to cis-elements and determines where

248 APOBEC1 performs C-to-U editing in mammalian systems [11]. Now that many non-PPR

249 editing factors have been discovered, there are evidently far more than 200 types of editosomes

250 in plant organelles.

To date, three families of proteins that lack PPR motifs have been identified as essential components of the plant editosome. Most of these factors affect many more editing targets than any individual PPR protein. Thus, most non-PPR factors are evidently present in a larger number

254 of editosomes than a single PPR protein, reflected by their greater expression levels compared to 255 PPR proteins. For example, 77\% of the mitochondrial C targets require RIP1 in their 256 editosomes, while $26 \%$ and $19 \%$ require RIP3 and RIP8, respectively $[4,73]$. Why particular 257 editing factors associate with specific PPR proteins is not understood, though yeast two-hybrid, 258 bimolecular fluorescence complementation, and pull-down assays (Table 2) indicate that certain 259 interactions occur and others do not. One way of maintaining selective interaction is segregation 260 of editing factors into different organelles by targeting of import from the cytoplasm. Most 261 factors enter only the plastid or the mitochondrial compartments, though exceptions exist, such 262 as RIP1, which is found in both organelles [68].

263 Although loss of expression of orrm 1, ozl, and rip/morf gene expression can sometimes 264 cause complete absence of editing of certain sites, some family members are functionally 
redundant. Removing one factor often results in a partially reduced editing efficiency of

266 particular $\mathrm{C}$ targets, suggesting that other family members can fulfill editing functions in the

267 absence of another member. Direct evidence for this hypothesis comes from a study on two

268 mitochondrial factors ORRM2 and ORRM3, which share many cognate sites. While residual

269 editing levels were observed in an orrm 3 mutant, silencing of ORRM 2 in the orrm 3 mutant

270 further reduced the editing efficiency [72]. ORRM2 and ORRM3 form heterodimers in yeast

271 two-hybrid assays, and ORRM3 can form a homodimer [72]. Possibly, an editosome carrying

272 ORRM3 homodimer can substitute for an ORRM2-ORRM3 heterodimer when ORRM2 is

273 missing, though the ensuing editosome is not as efficient or not sufficiently abundant.

274 A similar situation occurs in editing of plastid $n d h G$ C50, in which the editosome is

275 predicted to contain at least RIP9, ORRM1 and OZ1 and likely often RIP2 as well. No editing of

$276 n d h G$ C50 occurs when RIP9 is absent, while 40\% editing remains in the rip2 null mutant [73].

277 Possibly the $n d h G$ C50 editosomes are more efficient when they contain a heteromer of RIP2

278 and RIP9, but one without RIP2 still can operate (Figure 2). Alternatively, in the absence of

279 RIP2, there may be inadequate amounts of RIP9 for efficient editing of $n d h G$ C50. Some

280 evidence that RIP/MORF heteromers may sometimes associate with PPR proteins comes from an

281 experiment with mitochondrial factors. Expression of MORF8/RIP1 along with the PPR protein

282 MEF13 increased interaction of MEF13 and MORF3/RIP3 in a yeast 3-hybrid system [88].

\section{Concluding Remarks and Future Perspectives}

285 Unlike its counterparts in mammals, the plant editosome varies at most editing sites, not 286 only in the PPR site-specific recognition factor, but also in the non-PPR factors (Figure 3, Key 287 Figure). For some sites whose editosome components from each of the four families have been 288 identified, the combined size of all factors, postulating each as a monomer, is at the low end of 289 the observed size of editosome in size exclusion chromatography ( 200kDa). As some of the 290 proteins may be present as multimers, possibly all of the proteins present in such editosomes 291 have been identified, which thus awaits experimental confirmation through reconstitution of the 292 editing complex in vitro. Alternatively, there might still be more unknown editing factors for 293 some, or all sites (see also "Outstanding Questions"). A number of ORRM and OZ family 294 members still remain uncharacterized. There are also "orphan sites" whose editing extents are 295 not substantially affected in any rip, orrm, or oz mutants or in tissue silenced for a known editing 
296 factor. These orphan sites could be ones that can be operated upon by many different editing

297 factors, so that redundancy prevents expression of a mutant phenotype — or they could be sites

298 for which some actual editing factors have not yet been identified.

299 Why are plant editosomes so diverse in composition? Consider the plight of a plant

300 organelle whose DNA has undergone a T-to-C mutation. If the amino acid affected seriously

301 impairs the function of a protein, there would be strong selection for organelles that can

302 overcome the deficiency through RNA editing. Perhaps an existing PPR protein may exhibit

303 weak binding to cis-element near a new T-to-C mutation. Other organelle proteins that exhibit

304 chance binding to the PPR protein or the cis-element may be recruited to enhance the initial

305 inefficient editing. Further selection could strengthen these interactions, thus enlisting a variety

306 of organelle proteins in the mission to rescue the organelle from a detrimental mutation. The

307 unexpected diversity of plant RNA editosomes may reflect haphazard placement of temporary

308 bandages onto defective RNA encoded by damaged DNA.

309 While evolution created a complex and diverse set of editosomes in order to deal with

310 organelle DNA mutations, modern genetic engineers are not confined by selective constraints.

311 The PPR recognition code, along with a PPR protein structure [89], has provided insights into

312 designing proteins able to bind particular sequences. With further understanding of the minimal

313 requirements for the factors that must be requisitioned to supply PPR proteins with catalytic

314 activity, we should be able to design simpler editing complexes to direct editing events to targets

315 of our choice. With intelligent design, perhaps the unexpected complexity of plant RNA

316 editosomes will be shown to be unnecessary.

\section{Acknowledgements}

319 Recent work on RNA editing by the authors has been funded by the US National Science

320 Foundation, Division of Molecular and Cellular Biosciences, Genes and Genome Systems (grant

321 MCB-1330294 to M.R.H. and S.B., grant MCB-1020636 to S.B. and grant MCB-0929423 to

322 M.R.H.). We thank Xiaowen Shi for a critical reading of the manuscript.

324 References

325 1. Covello, P.S. and Gray, M.W. (1989) RNA editing in plant mitochondria. Nature 341, 662-

$326 \quad 666$ 
2. Gualberto, J.M., et al. (1989) RNA editing in wheat mitochondria results in the conservation

328 of protein sequences. Nature 341, 660-662

329 3. Hiesel, R., et al. (1989) RNA editing in plant mitochondria. Science 246, 1632-1634

330 4. Bentolila, S., et al. (2013) Comprehensive high-resolution analysis of the role of an

331 Arabidopsis gene family in RNA editing. PLoS Genet 9, e1003584

332 5. Stern, D.B., et al. (2010) Chloroplast RNA metabolism. Annual Review of Plant Biology 61, $333 \quad 125-155$

334 6. Oldenkott, B., et al. (2014) Chloroplast RNA editing going extreme: more than 3400 events of

335 C-to-U editing in the chloroplast transcriptome of the lycophyte Selaginella uncinata. RNA 20, $336 \quad 1499-1506$

337 7. Takenaka, M., et al. (2014) RNA editing in plant mitochondria-connecting RNA target 338 sequences and acting proteins. Mitochondrion $19 \mathrm{Pt} \mathrm{B}, 191-197$

339 8. Castandet, B. and Araya, A. (2011) RNA editing in plant organelles. Why make it easy?

340 Biochemistry Biokhimiia 76, 924-931

341 9. Bock, R. (2000) Sense from nonsense: how the genetic information of chloroplasts is altered

342 by RNA editing. Biochimie 82, 549-557

343 10. Chateigner-Boutin, A.L. and Small, I. (2010) Plant RNA editing. RNA biology 7, 213-219

344 11. Smith, H.C., et al. (1997) A guide to RNA editing. Rna 3, 1105-1123

345 12. Stern, D.B., et al. (2004) Genetics and genomics of chloroplast biogenesis: maize as a model 346 system. Trends in Plant Science 9, 293-301

347 13. Hanson, M.R., et al. (1996) Plant organelle gene expression: Altered by RNA editing. Trends

348 in Plant Science 1, 57-64

349 14. Shikanai, T. (2015) RNA editing in plants: Machinery and flexibility of site recognition.

350 Biochimica et biophysica acta 1847, 779-785

351 15. Sommer, B., et al. (1991) RNA editing in brain controls a determinant of ion flow in

352 glutamate-gated channels. Cell 67, 11-19

353 16. Teng, B., et al. (1993) Molecular cloning of an apolipoprotein B messenger RNA editing

354 protein. Science 260, 1816-1819

355 17. Mehta, A. and Driscoll, D.M. (1998) A sequence-specific RNA-binding protein complements 356 Apobec-1 to edit Apolipoprotein B mRNA. Molecular and Cellular Biology 18, 4426-4432 
18. Mehta, A., et al. (2000) Molecular cloning of apobec-1 complementation factor, a novel

358 RNA-binding protein involved in the editing of apolipoprotein B mRNA. Mol Cell Biol 20, $359 \quad 1846-1854$

360 19. Mehta, A. and Driscoll, D.M. (2002) Identification of domains in apobec-1 complementation 361 factor required for RNA binding and apolipoprotein-B mRNA editing. Rna 8, 69-82

362 20. Bass, B.L. and Weintraub, H. (1988) An unwinding activity that covalently modifies its 363 double-stranded RNA substrate. Cell 55, 1089-1098

364 21. Kim, U., et al. (1994) Molecular cloning of cDNA for double-stranded RNA adenosine 365 deaminase, a candidate enzyme for nuclear RNA editing. Proc. Natl. Acad. Sci. U.S.A. 91, $366 \quad 11457-11461$

367 22. Kim, U., et al. (1994) Purification and characterization of double-stranded RNA adenosine 368 deaminase from bovine nuclear extracts. J. Biol. Chem. 269, 13480-13489

369 23. Melcher, T., et al. (1996) A mammalian RNA editing enzyme. Nature 379, 460-464

370 24. Wagner, R.W., et al. (1989) A double-stranded RNA unwinding activity introduces structural 371 alterations by means of adenosine to inosine conversions in mammalian cells and Xenopus eggs.

372 Proc. Natl. Acad. Sci. U.S.A. 86, 2647-2651

37325 Gott, J.M. and Emeson, R.B. (2000) Functions and mechanisms of RNA editing. Ann.

374 Rev.Genetics 34, 499-531

375 26. Lu, B. and Hanson, M.R. (1994) A single homogeneous form of ATP6 protein accumulates 376 in petunia mitochondria despite the presence of differentially edited atp6 transcripts. The Plant 377 Cell 6, 1955-1968

378 27. Lu, B., et al. (1996) Protein polymorphism generated by differential RNA editing of a plant 379 mitochondrial rps12 gene. Mol Cell Biol 16, 1543-1549

380 28. Phreaner, C.G., et al. (1996) Incomplete editing of rps 12 transcripts results in the synthesis 381 of polymorphic polypeptides in plant mitochondria. Plant Cell 8, 107-117

38229 Tillich, M., et al. (2006) The evolution of chloroplast RNA editing. Mol. Biol. Evol. 23, 1912$383 \quad 1921$

38430 Fujii, S. and Small, I. (2011) The evolution of RNA editing and pentatricopeptide repeat 385 genes. New Phytol.191, 37-47

386 31. Guo, W., et al. (2016) Ginkgo and Welwitschia mitogenomes reveal extreme contrasts in 387 gymnosperm mitochondrial evolution. Mol. Biol. Evol., in press 
32 .Hein, A., et al. (2016) Frequent chloroplast RNA editing in early-branching flowering plants: pilot studies on angiosperm-wide coexistence of editing sites and their nuclear specificity factors. BMC Evol. Biol. 16, 23

33. Cheng, S., et al. (2016) Redefining the structural motifs that determine RNA binding and RNA editing by pentatricopeptide repeat proteins in land plants. Plant Journal 85, 532-547

34. Nishikura, K. (2010) Functions and regulation of RNA editing by ADAR deaminases. Ann. Rev. Biochem. 79, 321-349

35 Lurin, C., et al. (2004) Genome-wide analysis of Arabidopsis pentatricopeptide repeat proteins reveals their essential role in organelle biogenesis. Plant Cell 16, 2089-2103 36. Jinek, M., et al. (2012) A programmable dual-RNA-guided DNA endonuclease in adaptive bacterial immunity. Science 337, 816-821

37. Ishino, Y., et al. (1987) Nucleotide sequence of the iap gene, responsible for alkaline phosphatase isozyme conversion in Escherichia coli, and identification of the gene product. $J$.

401 Bact. 169, 5429-5433

402 38. Cong, L., et al. (2013) Multiplex genome engineering using CRISPR/Cas systems. Science $403339,819-823$

404 39. Montiel-Gonzalez, M.F., et al. (2013) Correction of mutations within the cystic fibrosis 405 transmembrane conductance regulator by site-directed RNA editing. Proc. Natl. Acad. Sci. U.S.A. 110, 18285-18290

407 40. Barkan, A., et al. (2012) A combinatorial amino acid code for RNA recognition by 408 pentatricopeptide repeat proteins. PLoS Genet 8, e1002910

409 41. Kindgren, P., et al. (2015) Predictable alteration of sequence recognition by RNA editing 410 factors from Arabidopsis. The Plant cell 27, 403-416

411 42. Coquille, S., et al. (2014) An artificial PPR scaffold for programmable RNA recognition.

412 Nature communications 5, 5729

413 43. Halter, C.P., et al. (2004) RNA editing in ribosome-less plastids of iojap maize. Current 414 genetics $45,331-337$

415 44. Zeltz, P., et al. (1993) Editing of the chloroplast rpoB transcript is independent of chloroplast 416 translation and shows different patterns in barley and maize. EMBO J 12, 4291-4296

417 45. Lightowlers, R.N. and Chrzanowska-Lightowlers, Z.M. (2013) Human pentatricopeptide 418 proteins: only a few and what do they do? RNA Biol. 10, 1433-1438 
46. Barkan, A. and Small, I. (2014) Pentatricopeptide repeat proteins in plants. Annu Rev Plant

420 Biol 65, 415-442

421 47. Takenaka, M., et al. (2013) Improved computational target site prediction for

422 pentatricopeptide repeat RNA editing factors. PLoS ONE 8, e65343

423 48. Yin, P., et al. (2013) Structural basis for the modular recognition of single-stranded RNA by

424 PPR proteins. Nature 504, 168-171

425 49. Hayes, M.L., et al. (2013) Identification of two pentatricopeptide repeat genes required for

426 RNA editing and zinc binding by C-terminal cytidine deaminase-like domains. J. Biol. Chem.

$427 \quad 288,36519-36529$

428 50. Okuda, K., et al. (2007) Conserved domain structure of pentatricopeptide repeat proteins

429 involved in chloroplast RNA editing. Proc. Natl. Acad. Sci. U.S.A. 104, 8178-8183

430 51. Okuda, K., et al. (2009) Pentatricopeptide repeat proteins with the DYW motif have distinct

431 molecular functions in RNA editing and RNA cleavage in Arabidopsis chloroplasts. Plant Cell

$432 \quad 21,146-156$

433 52. Salone, V., et al. (2007) A hypothesis on the identification of the editing enzyme in plant

434 organelles. FEBS Letters 581, 4132-4138

435 53. Schallenberg-Rudinger, M., et al. (2013) A survey of PPR proteins identifies DYW domains

436 like those of land plant RNA editing factors in diverse eukaryotes. RNA biology 10, 1549-1556

437 54. Hayes, M.L. and Hanson, M.R. (2007) Assay of editing of exogenous RNAs in chloroplast

438 extracts of Arabidopsis, maize, pea, and tobacco. Methods in enzymology 424, 459-482

439 55. Hegeman, C.E., et al. (2005) Substrate and cofactor requirements for RNA editing of

440 chloroplast transcripts in Arabidopsis in vitro. The Plant Journal 42, 124-132

441 56. Hirose, T. and Sugiura, M. (2001) Involvement of a site-specific trans-acting factor and a

442 common RNA-binding protein in the editing of chloroplast mRNAs: development of a

443 chloroplast in vitro RNA editing system. The EMBO Journal 20, 1144-1152

444 57. Takenaka, M. and Brennicke, A. (2003) In vitro RNA editing in pea mitochondria requires

445 NTP or dNTP, suggesting involvement of an RNA helicase. The Journal of biological chemistry

$446 \quad 278,47526-47533$

447 58. Takenaka, M., et al. (2007) In vitro RNA editing in plant mitochondria does not require

448 added energy. FEBS Lett 581, 2743-2747 
59. Nakamura, T. and Sugita, M. (2008) A conserved DYW domain of the pentatricopeptide

450 repeat protein possesses a novel endoribonuclease activity. FEBS Letters 582, 4163-4168

451 60. Okuda, K., et al. (2010) The pentatricopeptide repeat protein OTP82 is required for RNA

452 editing of plastid $n d h B$ and $n d h G$ transcripts. The Plant journal : for cell and molecular biology

$45361,339-349$

454 61. Boussardon, C., et al. (2014) The cytidine deaminase signature HxE(x)n CxxC of DYW1

455 binds zinc and is necessary for RNA editing of ndhD-1. The New phytologist 203, 1090-1095

456 62. Cao, Z.L., et al. (2011) A point mutation in the pentatricopeptide repeat motif of the AtECB2

457 protein causes delayed chloroplast development. Journal of integrative plant biology 53, 258-

$458 \quad 269$

459 63. Robbins, J.C., et al. (2009) A comparative genomics approach identifies a PPR-DYW protein

460 that is essential for C-to-U editing of the Arabidopsis chloroplast accD transcript. Rna 15, 1142-

$461 \quad 1153$

462 64. Yu, Q.B., et al. (2009) AtECB2, a pentatricopeptide repeat protein, is required for chloroplast

463 transcript accD RNA editing and early chloroplast biogenesis in Arabidopsis thaliana. Plant

464 Journal 59, 1011-1023

465 65. Wagoner, J.A., et al. (2015) Cytidine deaminase motifs within the DYW domain of two

466 pentatricopeptide repeat-containing proteins are required for site-specific chloroplast RNA

467 editing. J. Biol. Chem. 290, 2957-2968

468 66. Hayes, M.L., et al. (2015) A conserved glutamate residue in the C-terminal deaminase

469 domain of pentatricopeptide repeat proteins is required for RNA editing activity. J. Biol. Chem.

$470 \quad 290,10136-10142$

471 67. Kotera, E., et al. (2005) A pentatricopeptide repeat protein is essential for RNA editing in

472 chloroplasts. Nature 433, 326-330

473 68. Bentolila, S., et al. (2012) RIP1, a member of an Arabidopsis protein family, interacts with

474 the protein RARE1 and broadly affects RNA editing. Proc. Natl. Acad. Sci. U.S.A. 109, E1453-

$475 \quad$ E1461

476 69. Sun, T., et al. (2013) An RNA recognition motif-containing protein is required for plastid

477 RNA editing in Arabidopsis and maize. Proc. Natl. Acad. Sci. U.S.A. 110, E1169-E1178

478 70. Sun, T., et al. (2015) A zinc finger motif-containing protein is essential for chloroplast RNA

479 editing. PLoS Genet 11, e1005028 
71. Shi, X., et al. (2016) RNA recognition motif-containing protein ORRM4 broadly affects

481 mitochondrial RNA editing and impacts plant development and flowering. Plant physiology 170,

482 294-309

483 72. Shi, X., et al. (2015) Two RNA recognition motif-containing proteins are plant mitochondrial 484 editing factors. Nuc. Acids Res. 43, 3814-3825

485 73. Takenaka, M., et al. (2012) Multiple organellar RNA editing factor (MORF) family proteins 486 are required for RNA editing in mitochondria and plastids of plants. Proc. Natl. Acad. Sci. U.S.A. $487109,5104-5109$

488 74. Shi, X., et al. (2016) Organelle RNA recognition motif-containing (ORRM) proteins are 489 plastid and mitochondrial editing factors in Arabidopsis. Plant Signal. Behav., in press

490 75. Næsted, H., et al. (2004) Arabidopsis VARIEGATED 3 encodes a chloroplast-targeted, zinc491 finger protein required for chloroplast and palisade cell development. J. Cell Science 117, 48074924818

493 76. Zhang, F., et al. (2014) Tetrapyrrole biosynthetic enzyme protoporphyrinogen IX oxidase 1

494 is required for plastid RNA editing. Proc. Natl. Acad. Sci. U.S.A., 201316183

495 77. Coego, A., et al. (2005) An Arabidopsis homeodomain transcription factor,

496 OVEREXPRESSOR OF CATIONIC PEROXIDASE 3, mediates resistance to infection by

497 necrotrophic pathogens. Plant Cell 17, 2123-2137

498 78. Tillich, M., et al. (2009) Chloroplast ribonucleoprotein CP31A is required for editing and 499 stability of specific chloroplast mRNAs. Proc. Natl. Acad. Sci. U.S.A. 106, 6002-6007

500 79. Kupsch, C., et al. (2012) Arabidopsis chloroplast RNA binding proteins CP31A and CP29A 501 associate with large transcript pools and confer cold stress tolerance by influencing multiple 502 chloroplast RNA processing steps. Plant Cell 24, 4266-4280

503 80. Germain, A., et al. (2015) High-throughput quantification of chloroplast RNA editing extent 504 using multiplex RT-PCR mass spectrometry. Plant Journal 83, 546-554

505 81. Zehrmann, A., et al. (2015) Selective homo- and heteromer interactions between the Multiple 506 Organellar RNA Editing Factor (MORF) proteins in Arabidopsis thaliana. J. Biol. Chem. 290, $507 \quad 6445-6456$

508 82. Takenaka, M., et al. (2013) RNA editing in plants and its evolution. Ann. Rev. Genetics 47, $509 \quad 335-352$ 
510 83.. Vasudevan, A.A., et al. (2013) Structural features of antiviral DNA cytidine deaminases.

511 Biol. Chem. 394, 1357-1370

512 84. García-Andrade, J., et al. (2013) Mediated plastid RNA editing in plant immunity. PLoS

513 Pathog 9, e1003713

514 85. Yagi, Y., et al. (2013) Elucidation of the RNA recognition code for pentatricopeptide repeat

515 proteins involved in organelle RNA editing in plants. PLoS ONE 8, e57286

516 86. Gully, B.S., et al. (2015) The design and structural characterization of a synthetic

517 pentatricopeptide repeat protein. Acta Crystal. D, Biol. Crystal. 71, 196-208

518 87. Shen, C., et al. (2016) Structural basis for specific single-stranded RNA recognition by

519 designer pentatricopeptide repeat proteins. Nature Comm. 7, 11285

520 88. Glass, F., et al. (2015) MEF13 requires MORF3 and MORF8 for RNA editing at eight

521 targets in mitochondrial mRNAs in Arabidopsis thaliana. Mol. Plant 8, 1466-1477

522 89. Gully, B.S., et al. (2015) The solution structure of the pentatricopeptide repeat protein PPR10 523 upon binding atpH RNA. Nucl. Acids. Res. 43, 1918-1926

\section{Figure Legends}

527 Figure 1. Schematic representation of essential editing factors. (A) Arabidopsis has 528 approximately 193 editing-type PPR proteins. PPR proteins contain tandem PPR motifs and 529 additional C terminal domains - the E domain and DYW domain. E-type editing factors do not 530 have the DYW domain. DYW1 has a truncated E domain and a DYW domain. (B) Five RIP 531 proteins play a major role in editing. RIP proteins have a conserved motif at $\mathrm{N}$ termini while 532 RIP1 has an extended C terminus with unknown function. (C) To date, four ORRMs have been 533 shown to be editing factors. ORRM1 has two degenerate RIP motifs and one RRM motif while 534 other ORRMs do not have RIP motifs. ORRM3 and ORRM4 have glycine rich regions. (D) 535 There are four members in the OZ family and OZ1 is the only protein that has been studied in 536 RNA editing. OZ1 has a region at its $\mathrm{N}$ terminus that is conserved with other family members as well as two RanBP2 type zinc fingers.

539 Figure 2. Possible editosomes acting on $\boldsymbol{n d h} \boldsymbol{G}$ C50. The PPR protein OTP82 recognizes the 540 cis-element (pink line) 5' to the target C (red letter). Additional factors from the RIP, ORRM and 
541 OZ families are also required. Optimal editing efficiency ( $80 \%)$ is observed when both RIP2

542 and RIP9 are present. When there is no expression of RIP2, 40\% editing of $n d h G$ C50 remains,

543 thus RIP9 monomers or multimers may be able to partially substitute for RIP2. The actual

544 composition and stoichiometry of the subunits in an editosome is unknown, nor is it known

545 whether additional proteins are required.

547 Figure 3. Diversity of plant editosomes. The protein components of the editosome are nuclear-

548 encoded, cytosol-translated and translocated into each organelle. The diversity among plant

549 editosomes is depicted in, but not restricted to, the following examples. Shape variation indicates

550 different families, while color and pattern variation indicates different members within a family.

551 A question mark suggests possible involvement of a second PPR protein. 1) Members of non-

552 PPR families (RIP, ORRM, and OZ) are partially redundant. The major factor (solid circles) is

553 indispensable although another family member (dashed circles) may replace it with a reduced

554 activity. Mutation in a dispensable factor (dashed) leads to a minor editing defect, while loss of a

555 major factor (solid) causes major loss of editing. 2) Different members of non-PPR families are

556 fully redundant. Any component can be dispensable (dashed circles) since another family

557 member can be incorporated into the editosome. Loss of one factor will not cause severe editing

558 defects. 3) No redundancy. Editosome requires multiple copies of non-PPR factors. Loss of any

559 factor will severely affect editing efficiency. 4) The editosome contains unknown factors which

560 play equivalent roles to known non-PPR factors. The presence of OZ proteins in mitochondrial

561 editosomes is currently hypothetical. Printed with permission from a professional artist $\odot \mathrm{Li}$

562 Yao.

565 Glossary

566 ADAR: adenosine deaminase acting on RNA, an enzyme that binds to double-stranded RNA and 567 converts adenosine (A) to inosine (I).

568 APOBEC-1: apolipoprotein B mRNA editing enzyme, catalytic. The enzyme that carries out $569 a p o B$ editing as a homodimer in mammalian systems, in complex with other proteins. 
570 cis-element: The RNA sequence adjacent to the editing sites that can be recognized by the

571 editing machinery. It has been shown that the 15-20 nucleotides 5' to the editing sites are the

572 most critical cis-element and are specific targets of PPR proteins.

573 DYW domain: A highly conserved C-terminal domain in some PPR proteins. The domain

574 generally terminates with triplet amino acids Asp-Tyr-Trp (D-Y-W). This domain contains a

575 characteristic zinc binding motif (CXXC, HXE) found in cytidine deaminases that convert Cs to 576 Us.

577 Organelle: a specialized subunit within a cell that has a specific function. In plants, two types of 578 organelles - chloroplast and mitochondrion have their own genomes. Plant RNA editing occurs 579 in these two organelles.

580 OZ: Organelle Zinc finger proteins, a small protein family that are involved in plant RNA 581 editing. All members are targeted to organelles and share the conserved RanBP2 type zinc finger 582 motifs.

583 Pentatricopeptide Repeat (PPR): a 35 amino-acid degenerate repeat present in a number of 584 RNA-binding proteins.

585 PPO1: Protoporphyrinogen Oxidase 1, an enzyme required for tetrapyrrole metabolism 586 RNA editing: A form of RNA processing that makes discrete changes to specific nucleotide 587 sequences within an RNA molecule. Editing events can include insertion, deletion, or base 588 substitution of nucleotides. In higher plants Cytidine-to-Uridine editing occurs in chloroplast and 589 mitochondrial RNAs.

590 RNA editing factor Interacting Protein (RIP)/Multiple Organellar RNA editing Factor

591 (MORF): a small protein family based on sequence similarity. Several family members are 592 essential RNA editing factors. Different nomenclatures reflect their initial reporting in two 593 independent studies.

594 RNA editosome: The molecular machinery that conducts RNA editing. In plants, it is a small 595 protein complex that operates on specific editing sites. 
Table 1. List of identified non-PPR editing factors

\begin{tabular}{|c|c|c|c|c|c|c|c|}
\hline $\begin{array}{l}\text { Protein } \\
\text { family }\end{array}$ & Protein & Alias & $\begin{array}{l}\text { Mature } \\
\text { protein } \\
\text { size kDa }\end{array}$ & $\begin{array}{l}\text { Subcellular } \\
\text { localization }\end{array}$ & $\begin{array}{l}\% \text { affected } \\
\text { sites of all } \\
\text { chloroplast } \\
\text { sites }\end{array}$ & $\begin{array}{l}\% \text { affected } \\
\text { sites of all } \\
\text { mitochondrial } \\
\text { sites }\end{array}$ & Refs. \\
\hline \multirow[t]{5}{*}{ RIP/MORF } & RIP1 & MORF8 & 37 & Dual & $22 \%$ & $77 \%$ & {$[4,68]$} \\
\hline & RIP2 & MORF2; DAL & 19 & Chloroplast & $100 \%$ & NA & {$[4,73]$} \\
\hline & RIP3 & MORF3 & 23 & Mitochondrion & NA & $26 \%$ & {$[4,73]$} \\
\hline & RIP8 & MORF1 & 42 & Mitochondrion & NA & $19 \%$ & {$[4,73]$} \\
\hline & RIP9 & MORF9; DAG & 20 & Chloroplast & $97 \%$ & NA & {$[4,73]$} \\
\hline \multirow[t]{4}{*}{ ORRM } & ORRM1 & & 36 & Chloroplast & $62 \%$ & NA & [69] \\
\hline & ORRM2 & & 15 & Mitochondrion & NA & $6 \% \mathrm{~b}$ & [72] \\
\hline & ORRM3 & $\begin{array}{l}\text { GR-RBP3; At- } \\
\text { mRBP2b }\end{array}$ & 30 & Mitochondrion & NA & $19 \%$ & {$[72,74]$} \\
\hline & ORRM4 & $\begin{array}{l}\text { GR-RBP5; } \\
\text { At-mRBP2a }\end{array}$ & 29 & Mitochondrion & NA & $44 \%$ & [71] \\
\hline \multirow[t]{4}{*}{$\mathrm{OZ}$} & $\mathrm{OZ1}$ & VAR3 & 82 & Chloroplast & $81 \%$ & NA & {$[70,75]$} \\
\hline & PP01 & PPOX1 & 56 & Chloroplast & $50 \%$ & NA & [76] \\
\hline & OCP3 & & 32 & Chloroplast & $12 \%$ & NA & [77] \\
\hline & CP31 & & 26 & Chloroplast & $38 \%$ & NA & {$[78,79]$} \\
\hline
\end{tabular}

aMature protein is the protein without chloroplast/mitochondrial transit peptide. Sizes are predicted based on protein annotation in PPDB (ppdb.tc.cornell.edu)

bResults from silencing experiments instead of null mutants

NA: not applicable

Table 2. Protein-protein interaction matrix between known editing factors in each organelle

\begin{tabular}{|c|c|c|c|c|c|c|c|}
\hline & PPR factors & RIP2 & RIP9 & RIP1 & ORRM1 & OZ1 & PP01 \\
\hline \multicolumn{8}{|c|}{ Chloroplast } \\
\hline PPR factors & $\begin{array}{l}\text { Weak } \\
\text { interaction; } \\
\text { dimer in } \\
\text { crystal } \\
\text { structure }\end{array}$ & - & - & - & - & - & - \\
\hline RIP2 & Interaction & Homodimer & - & - & - & - & - \\
\hline RIP9 & Interaction & Heterodimer & Homodimer & - & - & - & - \\
\hline RIP1 & Interaction & Heterodimer & Heterodimer & Homodimer & - & - & - \\
\hline ORRM1 & Interaction & Interaction & Not tested & Interaction & No & - & - \\
\hline $\mathrm{OZ1}$ & Interaction & No & No & Weak & Interaction & Homodimer & \\
\hline PP01 & No & Interaction & Interaction & Interaction & Not tested & Not tested & Not tested \\
\hline \multicolumn{8}{|c|}{ Mitochondrion } \\
\hline PPR factors & Not tested & - & - & - & - & - & - \\
\hline RIP1 & Interaction & Homodimer & - & - & - & - & - \\
\hline RIP3 & Interaction & Heterodimer & Homodimer & - & - & - & - \\
\hline RIP8 & Interaction & Heterodimer & Heterodimer & Homodimer & - & - & - \\
\hline ORRM2 & No evidence ${ }^{a}$ & No & No & Not tested & No & - & - \\
\hline ORRM3 & No evidence ${ }^{a}$ & Interaction & No & Not tested & Heterodimer & Homodimer & - \\
\hline ORRM4 & No evidence ${ }^{a}$ & Interaction & Not tested & Not tested & No & Heterodimer & Homodimer \\
\hline
\end{tabular}


Number of genes in the family

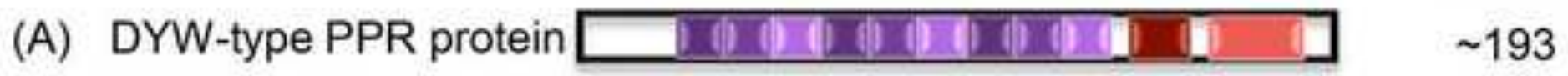

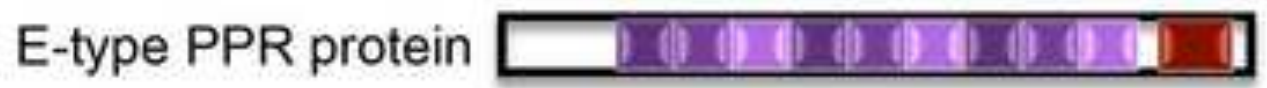

DYW-1 पII

(B)

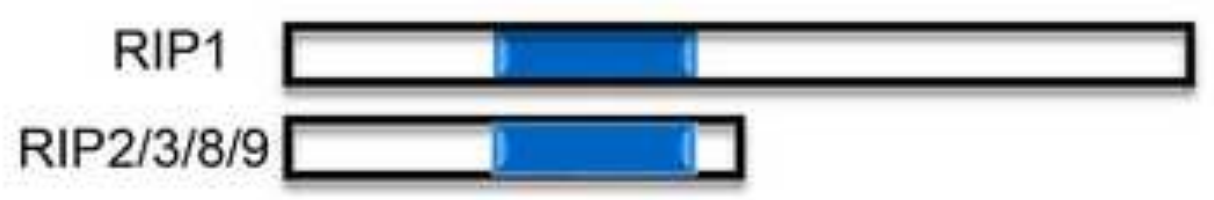

(C)

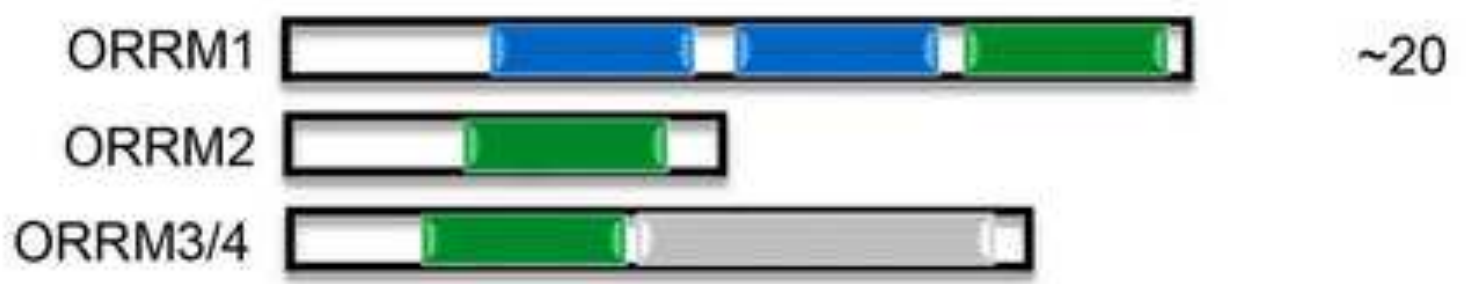

(D)

OZ1

4

\begin{tabular}{|c|c|c|c|}
\hline \multirow[t]{5}{*}{ Key: } & & & \\
\hline & [010 & PLS class PPR & III RRM \\
\hline & II & E domain & Dlycine rich domain \\
\hline & II & DYW & DZ conserved domain \\
\hline & [I & RIP/MORF motif & [1] Ran2BP zinc finger \\
\hline
\end{tabular}


(A) DYW-type PPR protein $\square$ | (|) (|) (1) (|) () () (|) () (I) (1)

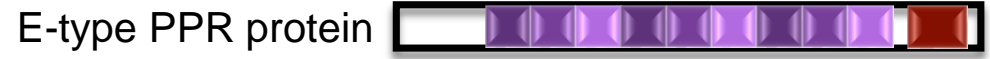

DYW-1 पाD

(B)

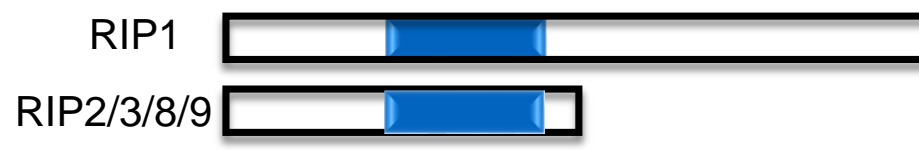

(C)

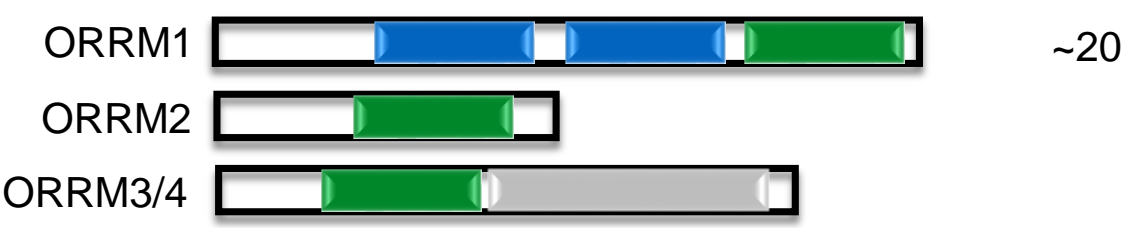

(D)

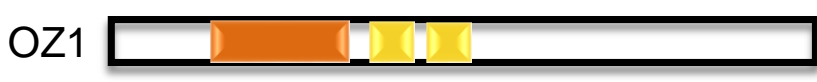

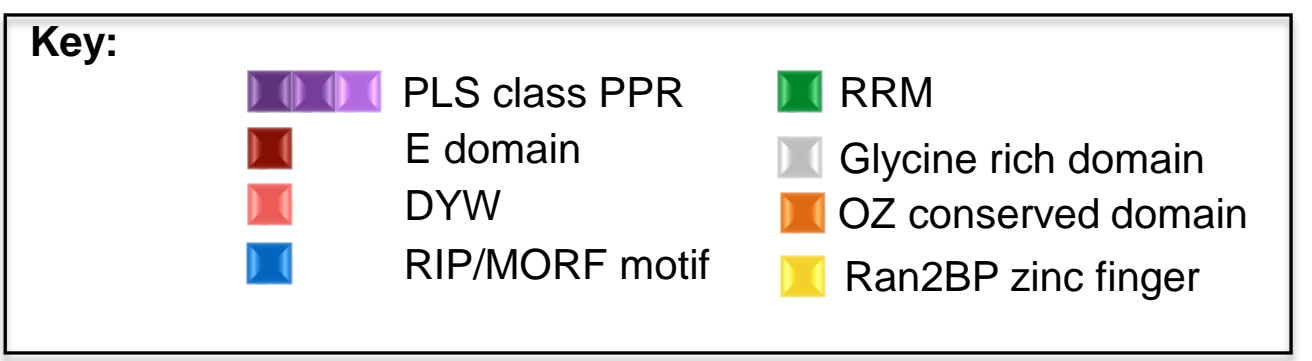




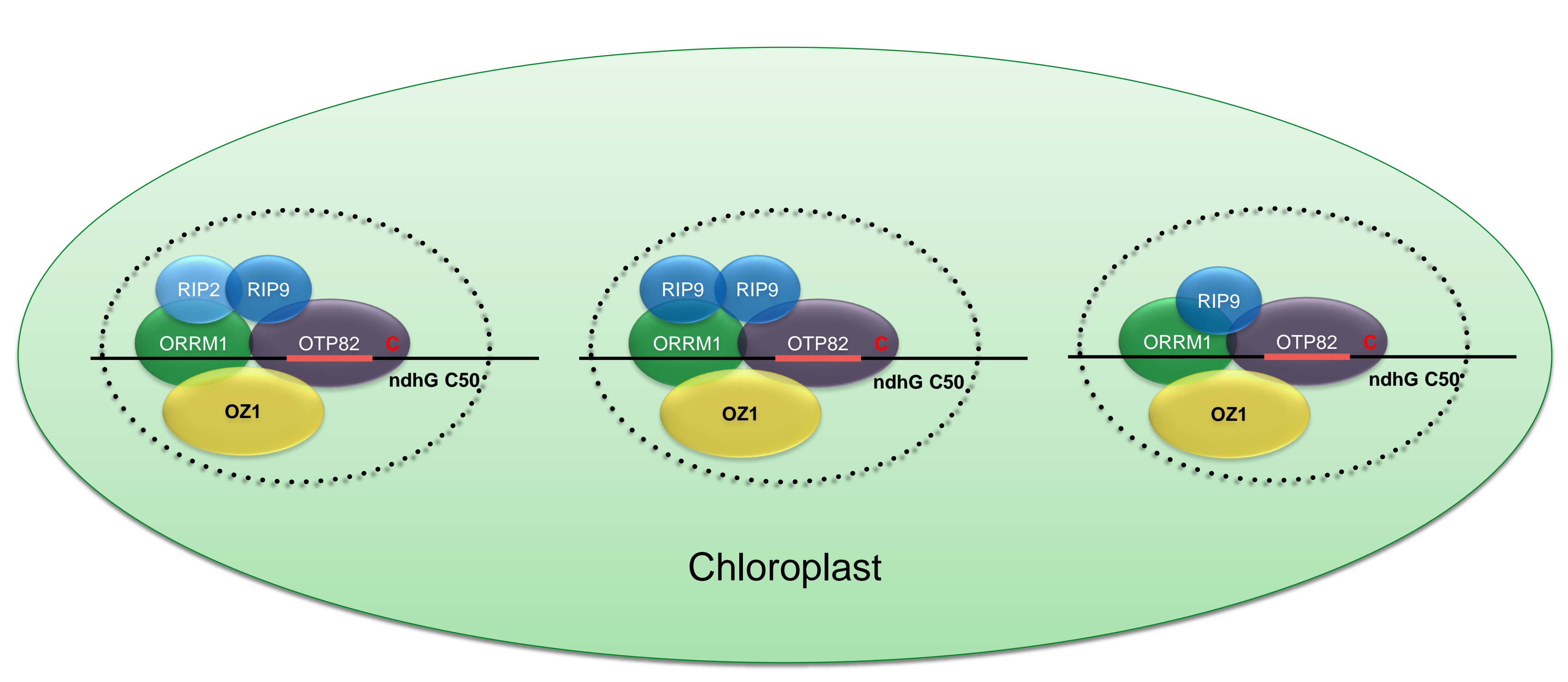




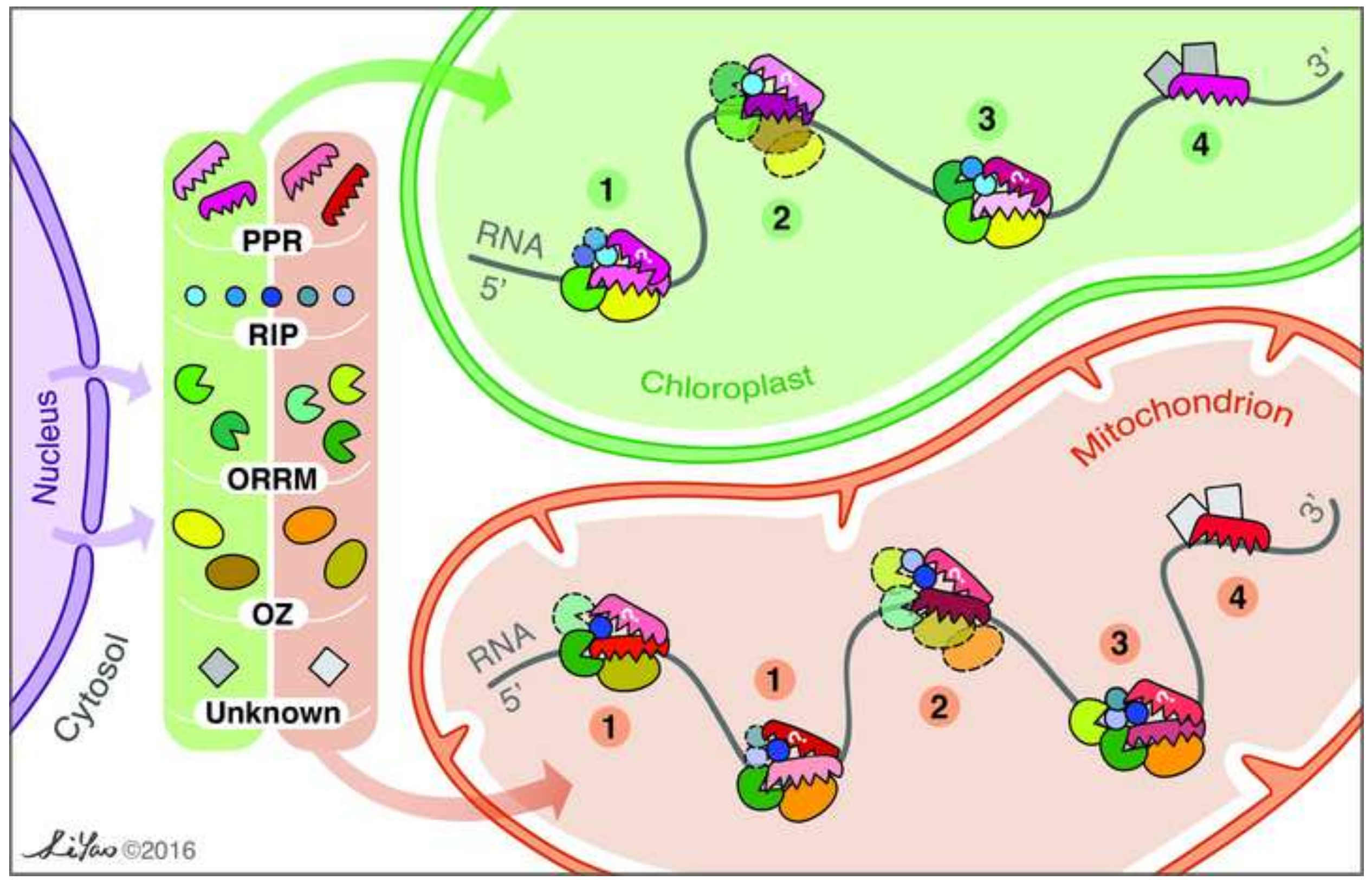

